\title{
Atorvastatin alters the expression of genes related to bile acid metabolism and circadian clock in livers of mice
}

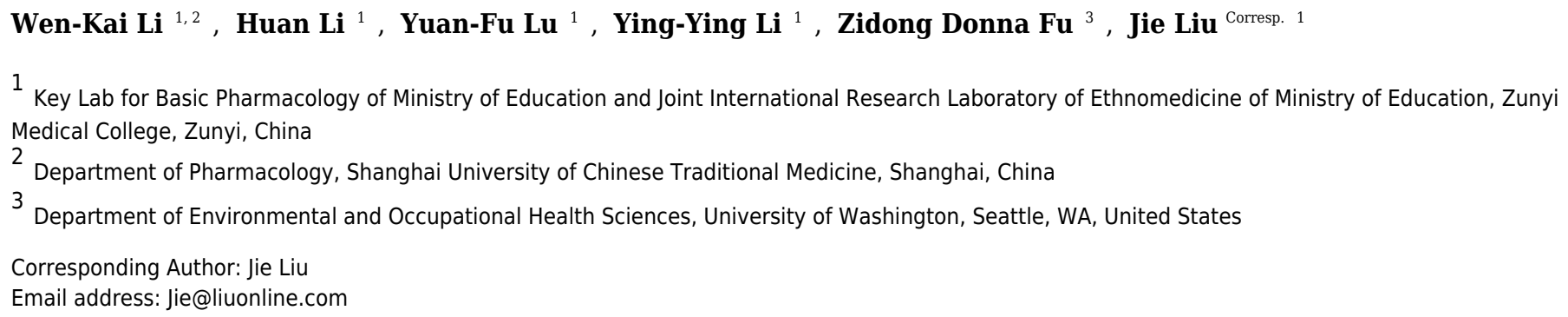

Aim: Atorvastatin is a HMG-CoA reductase inhibitor used for hyperlipidemia. Atorvastatin is generally safe but may induce cholestasis. The present study aimed to examine the effects of atorvastatin on hepatic gene expression related to bile acid metabolism and homeostasis, as well as the expression of circadian clock genes in livers of mice.

Methods: Adult male mice were given atorvastatin (10,30, and $100 \mathrm{mg} / \mathrm{kg}$, po) daily for 30 days, and blood biochemistry, histopathology, and gene expression were examined.

Results: Repeated administration of atorvastatin did not affect animal body weight gain or liver weights. Serum enzyme activities were in the normal range. Histologically, the high dose of atorvastatin produced scattered swollen hepatocytes, foci of feathery-like degeneration, together with increased expression of Egr-1 and metallothionein-1. Atorvastatin increased the expression of Cyp7al in the liver, along with FXR and SHP. In contract, atorvastatin decreased the expression of bile acid transporters Ntcp, Bsep, Ost $\alpha$, and Ost $\beta$. The most dramatic change was the 30-fold induction of Cyp7a1. Because Cyp7a1 is a circadian clock-controlled gene, we further examined the effect of atorvastatin on clock gene expression. Atorvastatin increased the expression of clock core master genes Bmal1 and Npas2, decreased the expression of clock feedback genes Per2, Per3, and the clock targeted genes Dbp and Tef, whereas it had no effect on Cryl and Nr1d1 expression. Conclusion: Repeated administration of atorvastatin affects bile acid metabolism and markedly increases the expression of the bile acid synthesis rate-limiting enzyme gene Cyp7a1, together with alterations in the expression of circadian clock genes. 
1 Atorvastatin alters the expression of genes related to bile acid metabolism and

2 circadian clock in livers of mice.

3 Wen-Kai Li ${ }^{1,2}$, Huan $\mathrm{Li}^{1}$, Yuan-Fu Lu ${ }^{1}$, Ying-Ying $\mathrm{Li}^{1}$, ZidongDonna Fü ${ }^{3}$, and Jie Liu ${ }^{1}$.

$4{ }^{1}$ Key Lab for Basic Pharmacology of Ministry of Education and Joint International

5 Research Laboratory of Ethnomedicine of Ministry of Education, Zunyi Medical

6 University, Zunyi, Guizhou, 563006, China

7 'Department of Pharmacology, Shanghai University of Traditional Chinese Medicine,

8 Shanghai 201203, China

$9{ }^{3}$ Department of Environmental and Occupational Health Sciences, University of

10 Washington, Seattle, WA 
11 Abstract

12 Aim: Atorvastatin is a HMG-CoA reductase inhibitor used for hyperlipidemia.

Atorvastatin is generally safe but may induce cholestasis. The present study aimed to examine the effects of atorvastatin on hepatic gene expression related to bile acid metabolism and homeostasis, as well as the expression of circadian clock genes in livers of mice.

Methods: Adult male mice were given atorvastatin (10, 30, and $100 \mathrm{mg} / \mathrm{kg}$, po) daily for 30 days, and blood biochemistry, histopathology, and gene expression were examined. Results: Repeated administration of atorvastatin did not affect animal body weight gain or liver weights. Serum enzyme activities were in the normal range. Histologically, the high dose of atorvastatin produced scattered swollen hepatocytes, foci of feathery-like degeneration, together with increased expression of Egr-1 and metallothionein-1. Atorvastatin increased the expression of Cyp7a1 in the liver, along with FXR and SHP. In contract, atorvastatin decreased the expression of bile acid transporters Ntcp, Bsep, Osta, and Ost $\beta$. The most dramatic change was the 30 -fold induction of Cyp7a1. Because Cyp7a1 is a circadian clock-controlled gene, we further examined the effect of atorvastatin on clock gene expression. Atorvastatin increased the expression of clock core master genes Bmal1 and Npas2, decreased the expression of clock feedback genes Per2, Per3, and the clock targeted genes Dbp and Tef, whereas it had no effect on Cry1 and Nr1d1 expression. 
31 Conclusion: Repeated administration of atorvastatin affects bile acid metabolism and markedly increases the expression of the bile acid synthesis rate-limiting enzyme gene Cyp7a1, together with alterations in the expression of circadian clock genes.

\section{Introduction}

Statins are the primary drugs clinically used to treat hyperlipidemia (Grover et al. 2014; Kalantari \& Naghipour 2014). As the number of hypercholesterolemic patients increases, statins, which are 3-hydroxy-3-methylglutaryl coenzyme A (HMG-CoA) reductase inhibitors, become more important. Statins are generally safe in clinical use (Kalantari \& Naghipour 2014), but there are few adverse drug reaction reports that are associated with statins, such as hepatotoxicity (Bjornsson et al. 2012; Bjornsson 2015; Grover et al. 2014; Kalantari \& Naghipour 2014).

Cholestasis is one of major adverse effects in statin-induced liver injury. It has been reported that atorvastatin induces prolonged cholestasis in patients (Beltowski et al. 2009; Merli et al. 2010). Accumulated bile acids could act as inflammagens, and directly activate signaling pathways in hepatocytes that stimulate production of proinflammatory mediators (Allen et al. 2011). It has been shown that the early growth response protein-1 (Egr-1) signal pathway and metallothionein-1 (MT-1) are involved in acute intrahepatic cholesteric liver injury (Allen et al. 2011; Alscher et al. 2002; Ding et al. 2008; Sullivan et al. 2012). 
50 Cholestasis is related to disruption of bile acid (BA) homeostasis (Parker et al. 2013).

51 Hydrophobic BAs are potent inflammatory chemical that cause injury to liver (Chiang 52 2013). In vitro and in vivo studies indicate that statins can alter BA metabolism in 53 hepatocytes (Byun et al. 2014; Fu et al. 2014). The rate-limiting enzyme for the classic 54 pathway of BA synthesis in the liver is Cyp7a1(Chiang 2013). It has been reported that 55 atorvastatin increases Cyp7a1, and alters the expression of Cyp7a1 regulatory genes, 56 farnesoid X receptor (FXR) and small heterodimer partner (SHP) (Byun et al. 2014; Fu 57 et al. 2014). BA transporters also play pivotal roles in maintaining BA homeostasis. BAs 58 are excreted into the bile by the bile salt export pump (Bsep), and transported from the 59 liver into blood by multidrug resistance-associated protein 3 and 4 (Mrp3 and Mrp4), and 60 organic solute transporter $\alpha$ and $\beta$ dimer (Osta/ $\beta$ ) (Chiang 2013; Fu et al. 2014). The 61 organic anion transporting polypeptide (Oatp1b2) and $\mathrm{Na}^{+}$-dependent taurocholate cotransport peptide (Ntcp) are responsible for BA uptake into hepatocytes (Cheng et al. 2007; Chiang 2013; Csanaky et al. 2011). Thus, an understanding of the effects of statins on bile acid homeostasis could be important in predicting the mechanism of statin-induced cholestasis.

The rate-limiting enzyme for bile acid homeostasis is Cyp7a1, which converts cholesterol to 7a-hydroxcholesterol (Chiang 2013). Statins have also been shown to influence the expression of Cyp7a1 (Fu et al. 2014; Jiang et al. 2012; Kolouchova et al. 2011). In one of our recent studies, atovarstatin at $100 \mathrm{mg} / \mathrm{kg}$, po for 7 days, induced Cyp7a1 (Fu et al., 2014), and whether such effects can be observed at lower doses and 
71 longer-times is our primary goals. Cyp7a1 is a circadian clock-driven gene displaying a

72 typical circadian rhythm (Kovar et al. 2010; Zhang et al. 2011), with a peak/trough ratio

73 over 10 fold (Lu et al. 2013). It is known that circadian dysregulation disrupts bile acid

74 homeostasis (Ferrell \& Chiang 2015b; Ma et al. 2009), and diurnal variation of hepatic

75 cholesterol synthesis is driven primarily by varying the steady-state mRNA levels for

76 HMG-CoA reductase (Jurevics et al. 2000). However, little is known about the effects of

77 statins on circadian rhythm, and this study is aimed to fill the gap.

78 Therefore, the goals of the present study are two-fold: (1) to examine the effect of

79 repeated administration of atorvastatin on bile acid homeostasis and cholestasis-

80 associated inflammation; and (2) to examine the effects of atorvastatin on the

81 expression of Cyp7a1 and circadian clock genes. The results demonstrate that

82 atorvastatin affects bile acid homeostasis, producing about 30-fold induction of Cyp7a1,

83 and this effect is associated with dysregulation of circadian clock genes.

\section{Materials and Methods:}

\section{Animals and treatment}

Adult male Kunming mice were obtained from the Experimental Animal Center of the Third Military Medical University (Chongqing, China) and randomly assigned to four groups. Mice were maintained in a room at $22 \pm 2^{\circ} \mathrm{C}$ with a $12 \mathrm{~h}$ light-dark cycle, and had free access to standard rodent chow and water. The control group of mice $(n=4)$ 
90 received saline and the treated groups ( $n=5 /$ per group) were given atorvastatin (Topfond

91 Pharmaceutical Co., Ltd, Henan, China) orally at doses of 10, 30 and $100 \mathrm{mg} / \mathrm{kg}$ once

92 daily for one month. The dose selection was based on human-mice dose conversion

93 (about $10 \mathrm{mg} / \mathrm{kg}, \mathrm{po}$ ), repeated doses in dog (up to $30 \mathrm{mg} / \mathrm{kg}$, po for 13 weeks) (Herron

94 et al. 2015) and the dose (100 mg/kg, po for 7 days) used for Cyp7a1 induction (Fu et al.

95 2014). The animal body weights were recorded every three days. In the morning at

$9610: 00$ am (1 $\mathrm{h}$ after the last dose of atovarstatin), the mice were anesthetized with $7 \%$

97 chloral hydrate, and blood and livers were collected. All animal experiments were carried

98 out in full compliance with the Guidance of Humane Care and Use of Laboratory

99 Animals, and approved by the Animal Care and Use Committee of Zunyi Medical 100 College (2013-5).

\section{Blood biochemistry}

102 The blood was kept at room temperature for one hour, and serum was separated by 103 centrifugation. Serum levels of alanine aminotransferase (ALT) were measured using a 104 commercial kit (Jiangcheng Co, Nanjing, China).

Histopathological procedure

Liver tissues were placed in plastic cassettes and immersed in $10 \%$ formalin for $48 \mathrm{~h}$. The fixed tissues were processed by standard histology procedures and subjected to hematoxylin and eosin (HE) staining. The degree of hepatocellular damage was evaluated by light microscopy. 
110 RNA isolation and RT-qPCR

111 Approximately $50-100 \mathrm{mg}$ of liver tissue was homogenized in $1 \mathrm{ml}$ Trizol (TakaRa

112 Biotechnology, Dalian, China) - The quality and quantity of RNA were determined by

113 the $260 / 280$ ratio (> 1.8) and by gel-electrophoresis. Total RNA was reversely

114 transcribed with a High Capacity Reverse Transcriptase Kit (Applied

115 Biosystems, Foster City, CA, USA) - The primers were designed with Primer3

116 software and listed in Supplemental Table 1 . The $15 \mu \mathrm{l}$ PCR reaction mix contained $3 \mu \mathrm{l}$

117 of cDNA $(10 \mathrm{ng} / \mu \mathrm{l}), 7.5 \mu \mathrm{l}$ of $\mathrm{iQ}^{\mathrm{TM}}$ SYBR GreenSupermix (Bio-Rad Laboratories,

118 Hercules, CA), $0.5 \mu \mathrm{l}$ of primer mix (10 $\mu \mathrm{M}$ each), and $4 \mu \mathrm{l}$ of $\mathrm{ddH}_{2} \mathrm{O}$. After 5 min

119 denature at $95^{\circ} \mathrm{C}, 40$ cycles will be performed: annealing and extension at $60^{\circ} \mathrm{C}$ for 45

120 seconds and denature at $95^{\circ} \mathrm{C}$ for 10 seconds. Dissociation curve was performed after

121 finishing 40 cycles to verify the quality of primers and amplification. Relative expression

122 of genes was calculated by the $2^{-\Delta \Delta \mathrm{Ct}}$-method and normalized to the housekeeping gene

$123 \beta$-actin.

124 Supplement table 1 Primer sequence for real-time RT-PCR analysis.

\begin{tabular}{llll}
\hline Gene & \multicolumn{1}{c}{$\begin{array}{c}\text { GenBank } \\
\text { Number }\end{array}$} & & Reverse (5' - 3') \\
\hline -actin & M12481 & GGCCAACCGTGAAAAGATGA & CAGCCTGGATGGCTACGTACA \\
Bmal1 & NM_007489 & ACGACATAGGACACCTCGCAGA & CGGGTTCATGAAACTGAACCATC \\
Bsep & NM_021022 & GGACAATGATGTGCTTGTGG & CACACAAAGCCCCTACCAGT \\
Cry1 & NM_007771 & GGATCCACCATTTAGCCAGACAC & CATTTATGCTCCAATCTGCATCAAG \\
Cyp7a1 & NM_007824 & ATCCTGGCAAACAGAAATCG & GGCCAAGTCTGGTTTCTCTG
\end{tabular}




\begin{tabular}{llll} 
Dbp & NM_016974 & ATCTCGCCCTGTCAAGCATTC & TGTACCTCCGGCTCCAGTACTTC \\
Egr-1 & NM_031332 & GTCTCTCTCTGGCCTGGTTT & GCACAAAGATGAGGGCCAAA \\
FXR & NM_009108 & TGGGTACCAGGGAGAGACTG & GTGAGCGCGTTGTAGTGGTA \\
MT-1 & NM_013602 & CTCCGTAGCTCCAGCTTCAC & AGGAGCAGCAGCTCTTCTTG \\
Nr1d1 & NM_145434 & GTGAAGACATGACGACCCTGGA & TGCCATTGGAGCTGTCACTGTAG \\
Npas2 & MA151656 & TGCTCCGAGAATCGAATGTGATA & ATGGCAGGCTGCTCAGTGAA \\
Ntcp & NM_011387 & GGTGCCCTACAAAGGCATTA & ACAGCCACAGAGAGGGAGAA \\
Ost $\alpha$ & NM_145932 & TTGTGATCAACCGCATTTGT & TTGTGATCAACCGCATTTGT \\
Ost $\beta$ & NM_178933 & ATCCTGGCAAACAGAAATCG & GGCCAAGTCTGGTTTCTCTG \\
Per2 & NM_011066 & CCTACAGCATGGAGCAGGTTGA & TTCCCAGAAACCAGGGACACA \\
Per3 & MA164628 & CTCAAGACGTGAGGGCGTTCTA & GGTTTCGCTGGTGCACATTC \\
SHP & NM_011850 & CTCATGGCCTCTACCCTCAA & GGTCACCTCAGCAAAAGCAT \\
Tef & MA032354 & CTTCAACCCTCGGAAGCACA & CCGGATGGTGATCTGGTTCTC \\
\hline
\end{tabular}

\section{Statistical analysis}

126 Data were expressed as mean and standard error. The SPSS 16 software was used for 127 statistical analysis. Data were analyzed using a one-way analysis of variance (ANOVA), 128 followed by Duncan's multiple range test. $p$ value $<0.05$ was considered statistically 129 significant.

\section{Results}

\section{Animal body weight, liver index and serum ALT levels}

132 Repeated administration of atorvastatin did not affect animal body weight gain, and did 133 not alter animal activity and general health. The animal body weight gain in the group 134 treated with $100 \mathrm{mg} / \mathrm{kg}$ atorvastatin was slightly lower than other groups, but was not 135 significantly different. The liver weight relative to the body weight ratios (liver index) also 136 showed no significant differences among the four groups $(48 \pm 2.8,48 \pm 4.2,51 \pm 5.0$, 
137 and $49 \pm 2.1 \mathrm{mg}$ liver/g body weight for Control, 10,30 , and $100 \mathrm{mg} / \mathrm{kg}$ atorvastatin 138 groups, respectively). Serum ALT is a biomarker of liver injury. The serum levels of ALT 139 in atorvastatin-treated mice were in general not elevated. Serum ALT levels in mice 140 treated with $100 \mathrm{mg} / \mathrm{kg}$ atorvastatin were slightly higher than the control group, but were 141 not statistically significant and within the normal range $(48 \pm 8.2,48 \pm 14,33 \pm 5.4$, and $14277 \pm 14 \mathrm{U} / \mathrm{L}$ for Control, 10, 30, and $100 \mathrm{mg} / \mathrm{kg}$ atorvastatin groups, respectively).

\section{2.Histopathological findings}

144 Liver sections from mice administered the various dosages of atorvastatin were stained 145 with H\&E and representative photographs are shown in Fig 1. There were no significant 146 differences between control and atorvastatin groups; however the foci of feathery-like 147 degeneration, indicative of mild cholestasis (think arrows) (Li \& Crawford 2004), were 148 observed. In mice treated with $100 \mathrm{mg} / \mathrm{kg}$ atorvastatin, spotted blood lakes 149 (peliosishepatis) can be seen (thick arrows). 

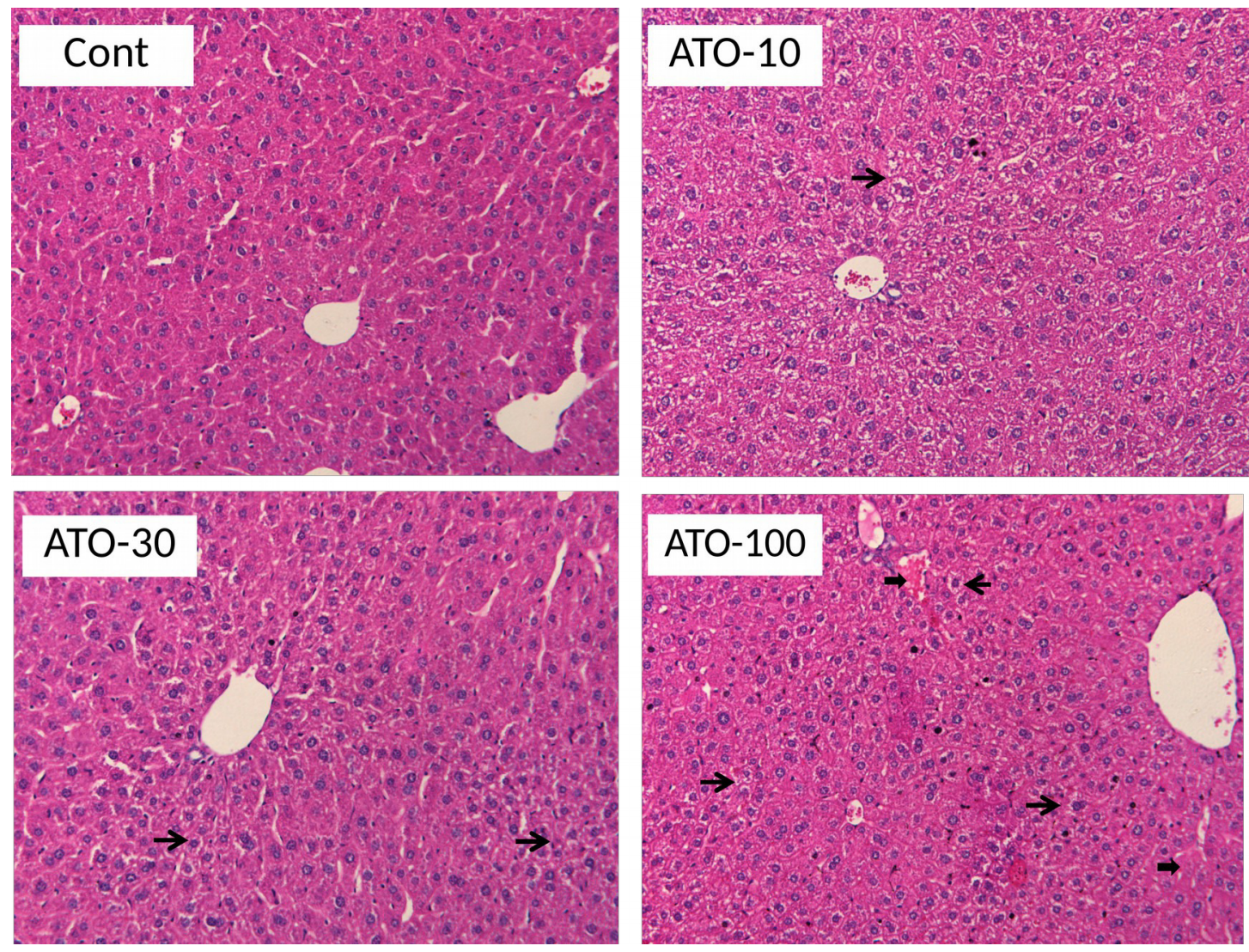

Fig. 1. Histopathology. Mice received atorvastatin 10,30 or $100 \mathrm{mg} / \mathrm{kg}$, po for 30 days. Representative H\&E staining of liver sections showed spotted feathery-like degeneration (thin arrows), and spotted blood lakes (peliosishepatis) (thick arrows). Magnitude $200 \mathrm{x}$.

\section{The expression of Egr-1 and MT-1 in liver}

154 Cholestasis contributes to liver injury and is associated with increased expression of 155 numerous proinflammatory mediators (Allen et al. 2011). In the present study, 156 atorvastatin significantly increased the expression of Egr-1 and MT-1, indicative of an 157 inflammatory response, in the livers of mice treated with atorvastatin for 30 days (Fig 2). 


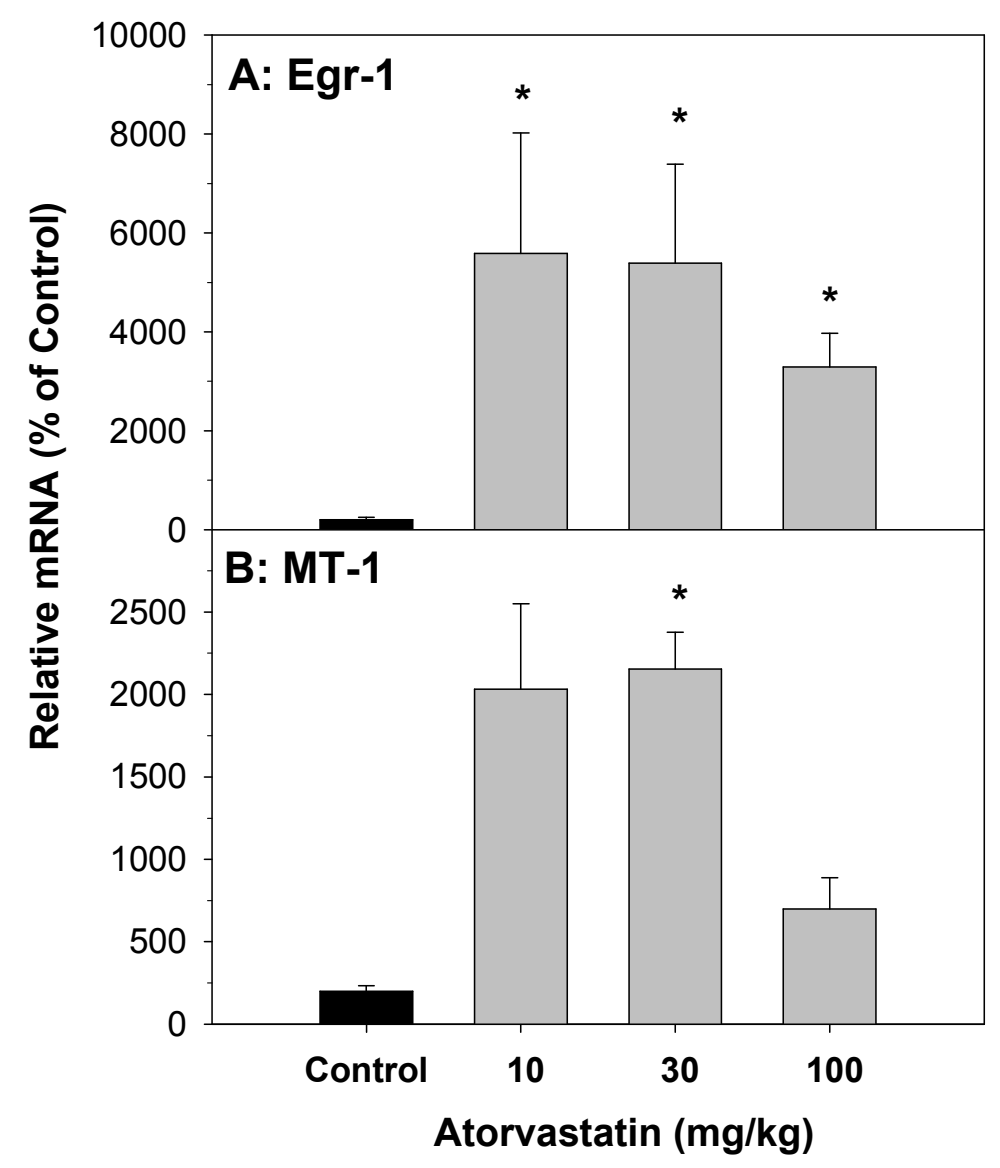

Fig. 2. Effects of atorvastatin treatment on mRNA expression of Egr-1 and MT-1 in mouse livers. Mice were given atorvastatin 10,30 or $100 \mathrm{mg} / \mathrm{kg}$, po for 30 days, Total liver RNA was extracted and subjected to RT-PCR analysis. Data represent the mean \pm SE of $n=5$. *Significantly different from control mice, $p<0.05$.

\section{The expression of genes related to BA-homeostasis}

Cyp7a1 encodes the rate-limiting enzyme for the classic pathway of BA synthesis in liver (Chiang 2013). The mRNA of Cyp7a1 in the atorvastatin-treated mice was markedly higher than in the control mice (26.1-fold at $10 \mathrm{mg} / \mathrm{kg}, 33.8$-fold at $30 \mathrm{mg} / \mathrm{kg}$, and 14 -fold at $100 \mathrm{mg} / \mathrm{kg}$ of atorvastatin). FXR is a bile acid-activated nuclear receptor. FXR 
167 induces transcription of the negative nuclear receptor, small heterodimer partner (SHP), 168 and SHP down-regulates Cyp7A1. In the present study, atorvastatin increased the 169 expression of FXR and SHP (Fig. 3).

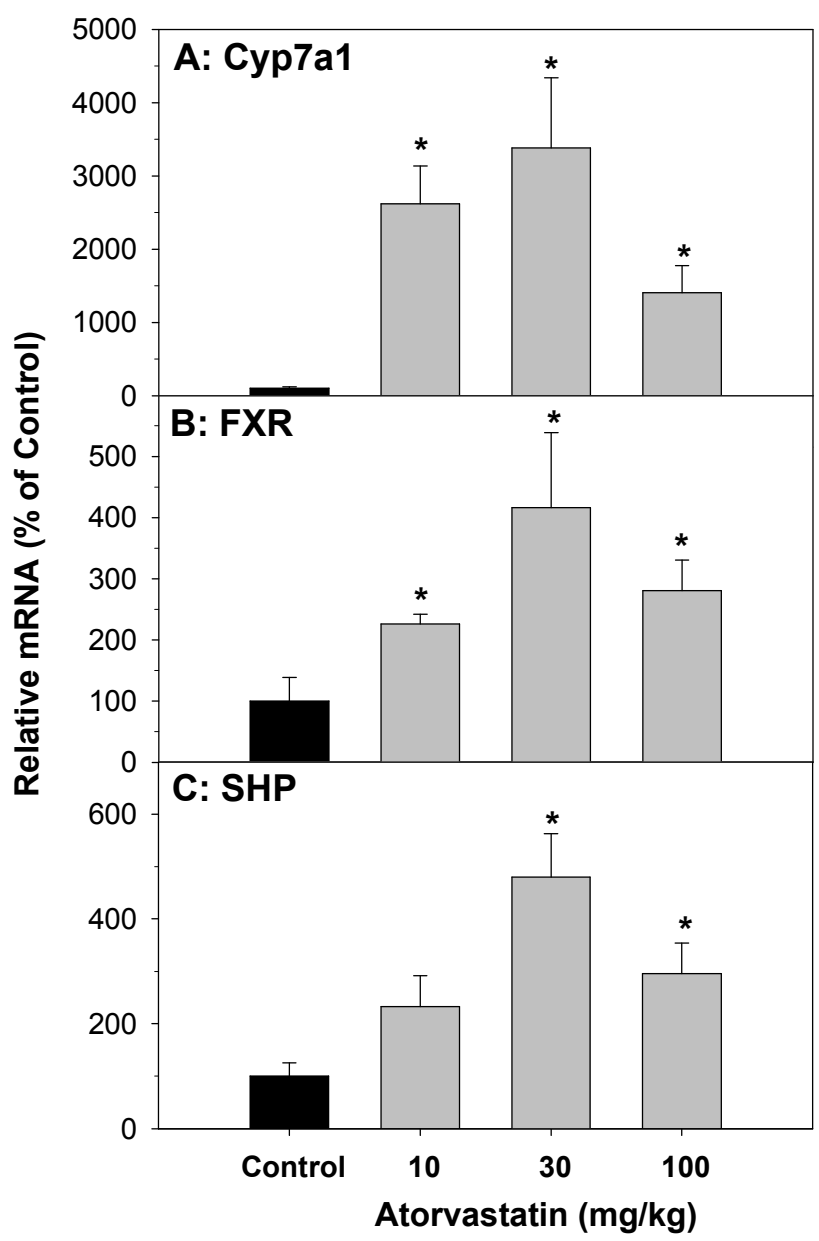

Fig. 3. Effects of atorvastatin treatment on mRNA expression of genes related to bile acid metabolism. Mice were given atorvastatin 10, 30 or $100 \mathrm{mg} / \mathrm{kg}$, po for 30 days. Total liver RNA was extracted and subjected to RT-PCR analysis. Data represent the mean \pm SE of $n=5$. *Significantly

173 different from control mice, $p<0.05$.

\section{5. Effects of mRNA expression of bile acid transporters}

175 The major transporters of bile acids in the liver include Bsep, Ntcp, Ost- $\alpha$ and Ost- $\beta$ 
176 (Chiang 2013; Klaassen \& Aleksunes 2010). The mRNA of Bsep (45.8-78.7\% of 177 controls), Ntcp (55.3-70.8\% of controls), Ost- $\alpha$ (20.1-36.7\% of controls) and Ost- $\beta$ (28.9$17858.8 \%$ of controls) was decreased after atorvastatin treatment (Fig. 4).

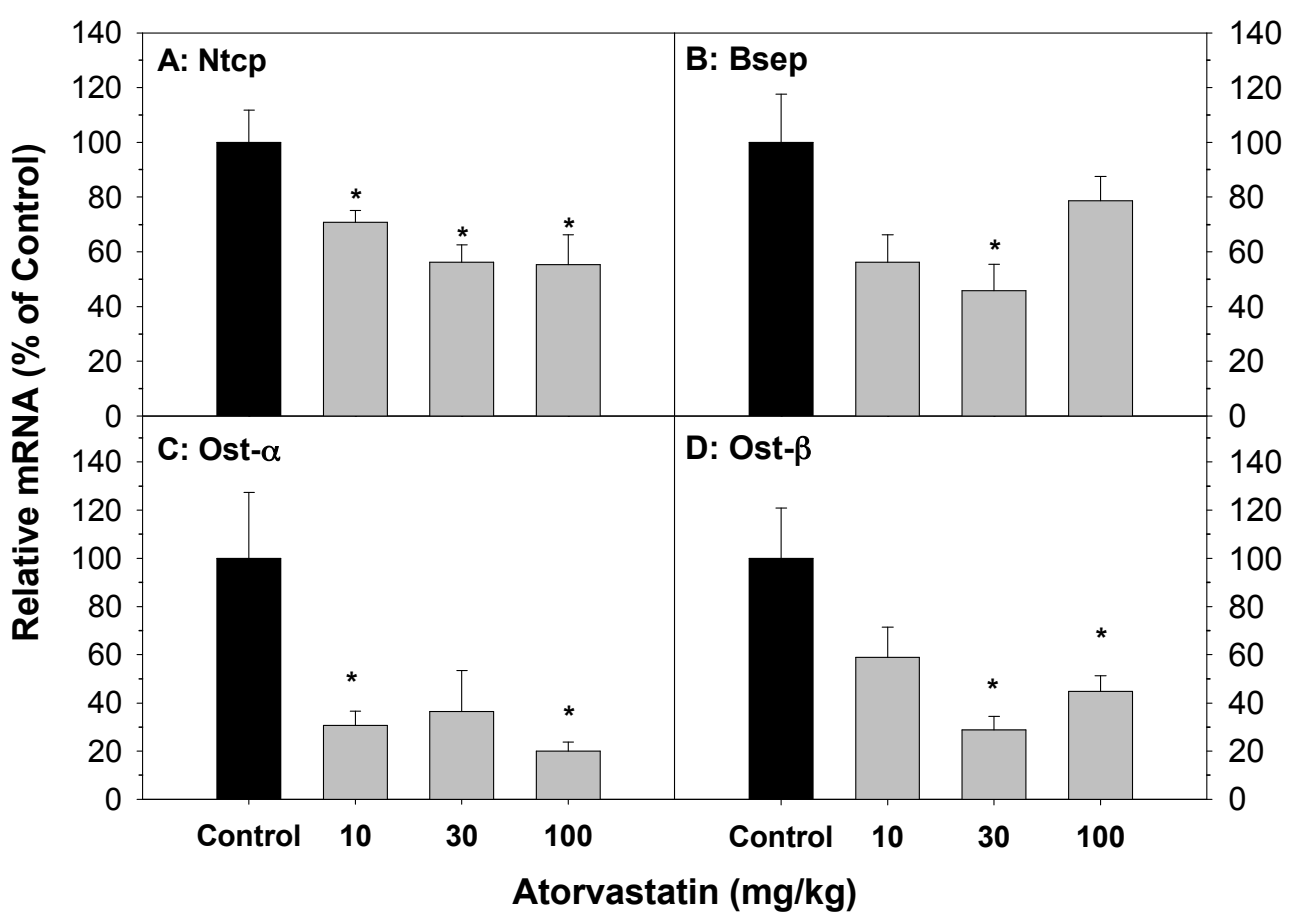

Fig. 4. Effects of atorvastatin treatment on mRNA expression of bile acid transporters. Mice were given atorvastatin 10,30 or $100 \mathrm{mg} / \mathrm{kg}$, po for 30 days. Total liver RNA was extracted and subjected to RT-PCR analysis. Data represent the mean \pm SE of $n=5$. *Significantly different from control mice, $p<0.05$.

\section{Effects of mRNA expression of circadian genes in liver}

Because the most dramatic effect of atorvastatin was the 33.8-fold induction of Cyp7a1, which is a clock-controlled gene (Lu et al. 2013), we further examined the effect of atorvastatin on the expression of clock genes. The Cyp7A1 gene promoter is under 
187 transcriptional control of the clock brain and muscle Arnt-like protein-1 (Bmal1) and other 188 clock genes (Noshiro et al. 2007). The mRNA expression of Bmal1 and neuronal PAS 189 domain protein 2 (Npas2) was increased by atorvastatin, in a similar trend as the 190 Cyp7a1 increases (Fig. 5).

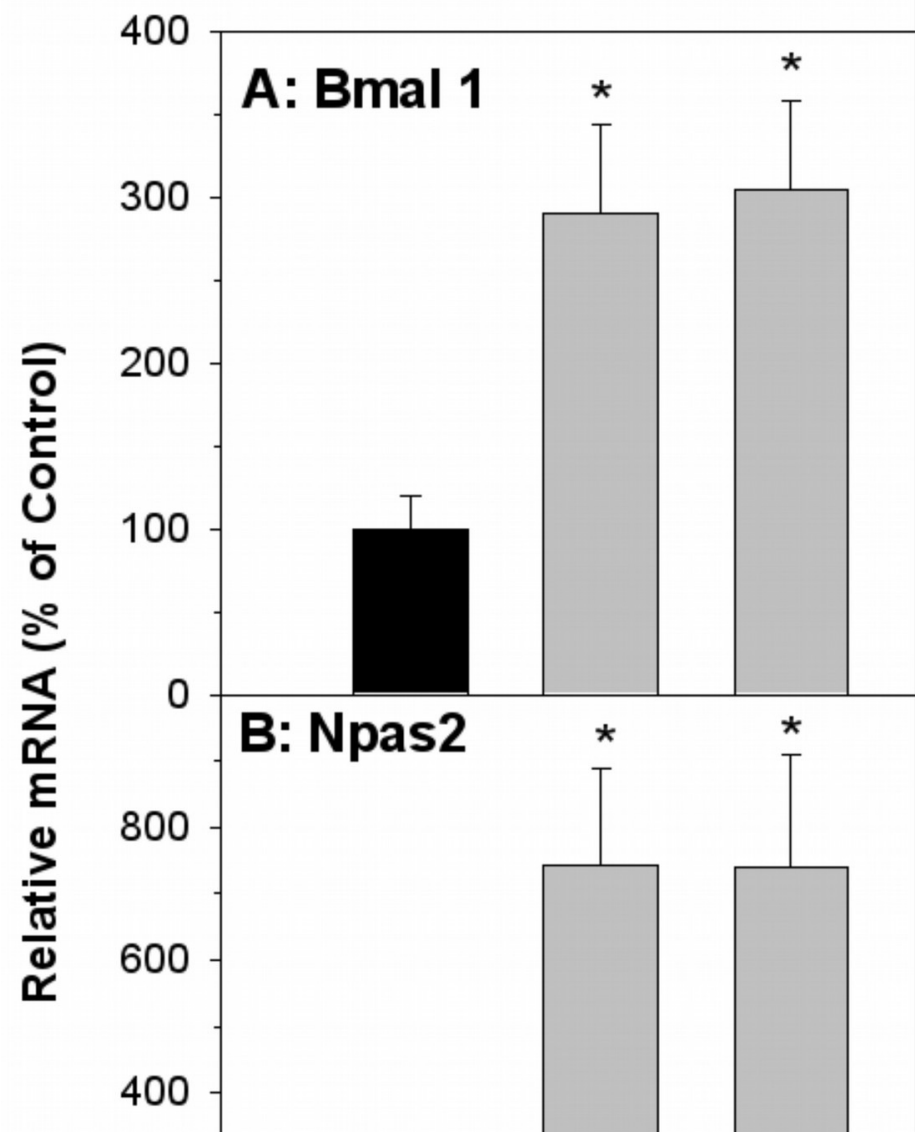

Fig 5. Effects of atorvastatin treatment on mRNA expression of clock core master genes Bmal1 and Npas2. Mice were administration atorvastatin 10,30 or $100 \mathrm{mg} / \mathrm{kg}$, po for 30 days, Total liver RNA was extracted and subjected to RT-PCR analysis. Data represent the mean \pm SE of 4-5 mice.

194 *Significantly different from control mice, $p<0.05$. 
195 For clock feedback control genes, atorvastatin decreased the expression of period 196 circadian protein gene 2 (Per 2) and Per 3, but had no effects on the mRNA expression 197 of cryptochrome 1 (Cry1) (Fig. 6).

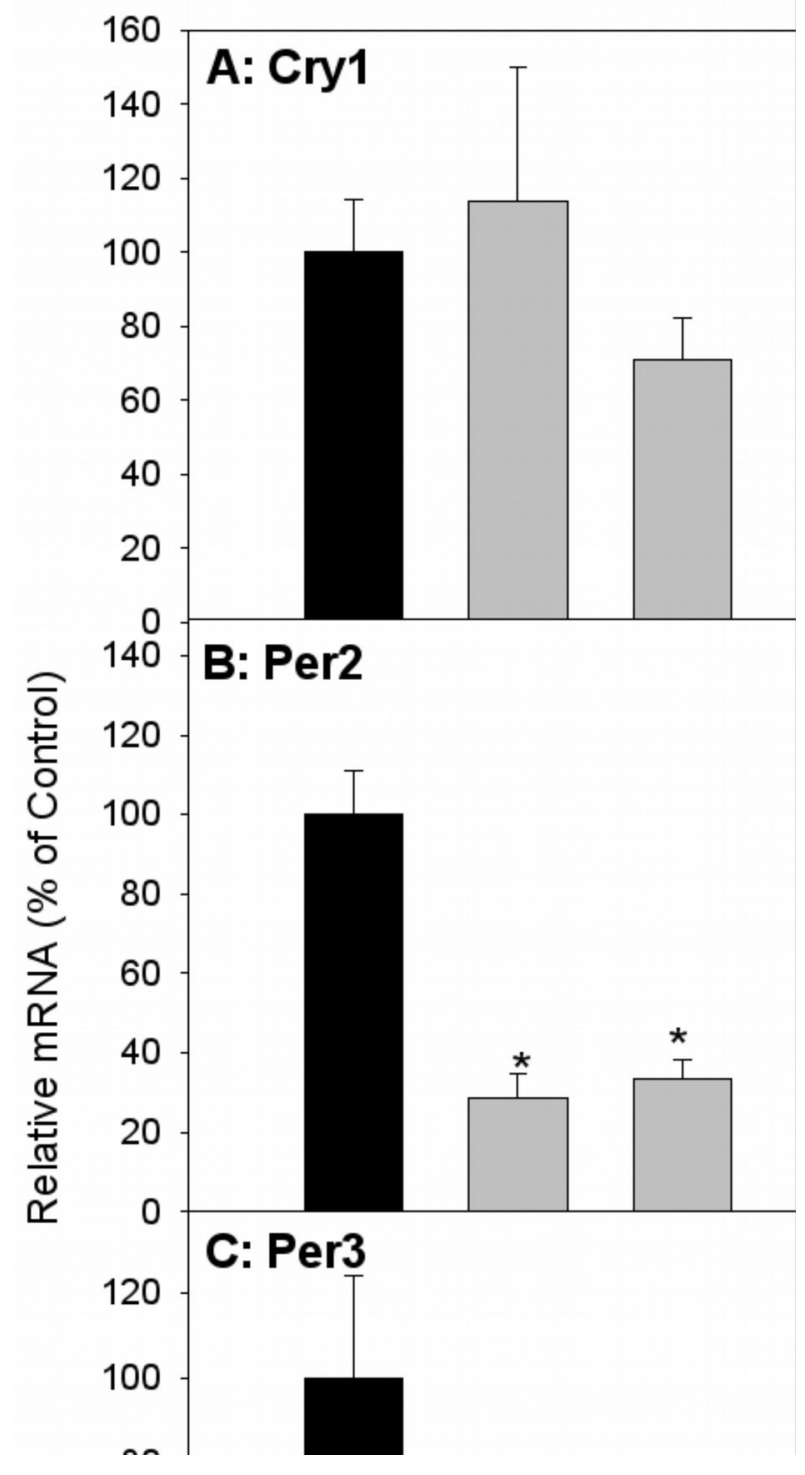

Fig 6. Effects of atorvastatin treatment on mRNA expression of clock feedback control genes Per2, 
200 was extracted and subjected to RT-PCR analysis. Data represent the mean \pm SE of $n=5$. 201 *Significantly different from control mice, $p<0.05$.

202 For clock-targeted/driven genes, the expression of $D$ site albumin promoter binding 203 protein (Dbp) and thyrotroph embryonic factor (Tef) in atorvastatin-treated mice was 204 significantly lower than that of the control group, while the expression of nuclear receptor 205 subfamily 1 , group $\mathrm{D}$, member 1 (Nr1d1) in atorvastatin-treated mice was unchanged 206 (Fig. 7). 


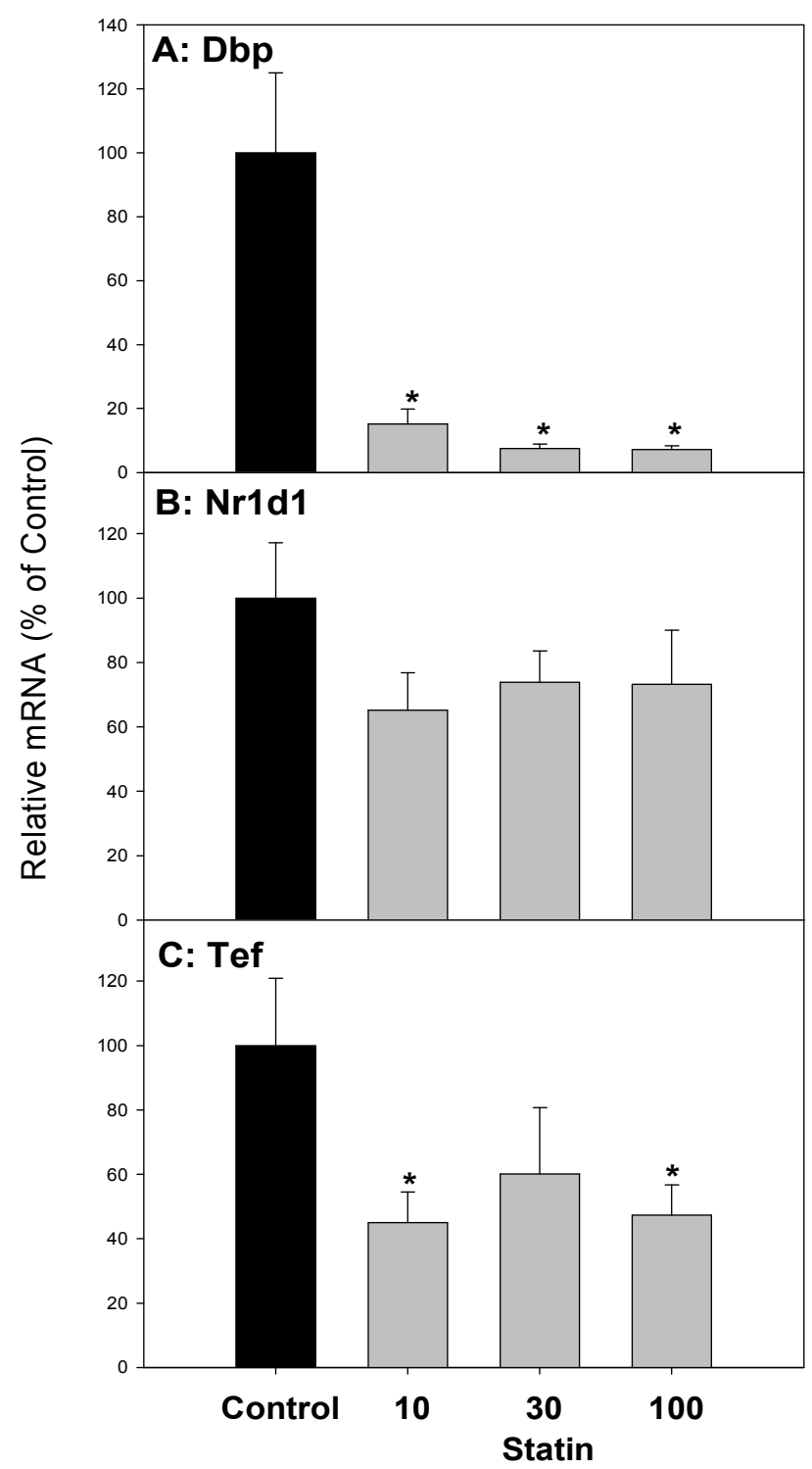

207 Fig 7. Effects of atorvastatin treatment on mRNA expression of clock targeted or driven genes Dbp, 208 Tef, and Nr1d1. Mice were given atorvastatin 10, 30 or $100 \mathrm{mg} / \mathrm{kg}$, po for 30 days, Total liver RNA 209 was extracted and subjected to RT-PCR analysis. Data represent the mean \pm SE of $n=5$. $210 *$ Significantly different from control mice, $p<0.05$. 
212 The present study showed that repeated administration of high doses of atorvastatin did 213 not alter animal body weight gain, liver weight, serum ALT, or cause overt pathological 214 alterations except for foci of inflammation, suggesting that atorvastatin is generally safe.

215 However, at these doses atorvastatin increased the gene expression of Cyp7a1 as 216 previously reported (Fu et al. 2014). Because Cyp7a1 is a clock-controlled gene (Lu et 217 al. 2013), we further examined the effects of atorvastatin on the expression of circadian 218 clock genes, and found Atorvastatin increased the expression of clock core master 219 genes Bmal1 and Npas2, decreased the expression of clock feedback genes Per2, 220 Per3, and the clock targeted genes Dbp and Tef in the livers of mice.

221 Few studies have shown that statins can induce oxidative stress, and elevate serum AST and ALT (Beltowski et al. 2009; Kolouchova et al. 2011). In the present study, no apparent liver toxicity was observed even after a high dose of atorvastatin $(100 \mathrm{mg} / \mathrm{kg}$ po for 30 days). This is in agreement with the majority of the literature that statins are relatively safe (Kalantari \& Naghipour 2014). However, the high dose of atorvastatin had a potential to produce cholestasis. Cholestasis is characterized by decreased bile flow resulting in accumulation of bile acids with spotted feathery-like degeneration in hepatocytes (Liu et al. 2013; Lu et al. 2013), and is also the main feature of statininduced liver injury (Kerzner et al. 2013; Merli et al. 2010; Russo et al. 2009). Overload of bile acids is a physiological stress that leads to inflammatory-associated gene expression, such as MT, a metal-binding, cysteine-rich protein (Alscher et al. 2002), as well as Egr-1, a critical regulator of inflammation and cellular stress (Kalantari \& 
233 Naghipour 2014; Kim et al. 2006; Russo et al. 2009). Overexpression of inflammatory 234 genes is accompanied by cholestasis. In the present study the expression of Egr1 was 235 significantly increased, and the expression of MT-1 was also increased, indicative of 236 cholestatic stress produced by atorvastatin.

237 Cholestasis increases the concentrations of bile acids in liver. Hepatic BA homeostasis 238 is tightly controlled by a variety of regulators within a relatively narrow range (Chiang 239 2013; Perez \& Briz 2009). It is known that both of BA synthetic enzymes and BA 240 transporters play an important role in BA homeostasis. The data of the present study 241 showed that atorvastatin significantly increased the expression of Cyp7a1, confirming 242 recent reports (Byun et al. 2014; Fu et al. 2014). The expression of Cyp7a1 is regulated 243 by a mechanism involving the BA-activated farnesoid $X$ receptor $(F X R)$ in hepatocytes 244 and enterocytes. Activation of FXR induces small heterodimer partner (SHP) to 245 feedback inhibit Cyp7a1 gene transcription in liver (Fu et al., 2014). The present study 246 shows that atorvastatin increased the expression of FXR and SHP similarly as Cyp7a1 247 (Fig 4). The increased expression in SHP in the present study is unexpected compared 248 to the literature (Fu et al. 2014). This discrepancy could be due to the long-term 249 administration of atovarstatin (30 vs 7 days), and another mechanism might be involved.

250 Accumulation of bile acids in the liver is associated with alterations in bile acid 251 transporters (Fu et al. 2014; Klaassen \& Aleksunes 2010; Liu et al. 2013). The retention 252 and accumulation of hydrophobic bile acids inside hepatocytes during cholestasis have 
253 long been implicated as a major cause of BA-induced hepatic injury (Perez \& Briz 2009)

254 and dysfunction of bile acid transporters. In the present study, atorvastatin decreased

255 the expression of the main conjugated BA uptake transporter Ntcp and the main BA

256 efflux transporter Bsep, but also decreased the expression of Osta/ $\beta$, which are BA

257 transporters in the liver and ileum (Fig 5). The decreased expression of bile acid 258 transporters (Bsep, Ost $\alpha / \beta)$ might contribute to the accumulation of bile acids in the liver 259 and stimulation of Cyp7a1 expression.

260 Circadian rhythms play important roles in liver metabolism and diseases including BA 261 metabolism and Cyp7a1(Ferrell \& Chiang 2015a). Cyp7a1 is a clock-driven gene 262 showing a typical circadian rhythm (Beltowski et al. 2009; Duez et al. 2008; Le Martelot 263 et al. 2009; Noshiro et al. 2007; Zhang et al. 2011). Circadian variation of hepatic 264 cholesterol synthesis is driven by HMG-CoA reductase (Jurevics et al. 2000), and HMG265 CoA inhibitor atorvastatin could likely affect the circadian clock. The mammalian 266 circadian clock is based on a transcription-translation feedback loop, which includes 267 core genes: CLOCK:BMAL1, Npas2:Bmal1, and feedback genes Per, Cry and 268 Nr1d1(Bozek et al. 2009; Ikeda \& Nomura 1997; Ye et al. 2014). Some of the clock269 controlled genes ( $\mathrm{Dbp}, \mathrm{Dec} 2$, and $\mathrm{Nr} 1 \mathrm{~d} 1)$ are direct regulators required for the robust 270 circadian rhythm of Cyp7a1(Noshiro et al. 2007). Furthermore, SHP, the regulator of 271 Cyp7a1, also has proved to be a downstream target of circadian clock (Pan et al. 2010). 272 It has been observed that the dysregulation of Bmal1 and Npas2 in SHP-null mice (Lee 273 et al. 2015; Wu et al. 2016). The present study shows that atorvastatin-induced increase 
274 in Cyp7a1 and SHP are associated with the core-clock dysregulation: Bmal1 and Npas2 275 were up-regulated after atorvastatin-treatment similar to induction of Cyp7a1. In 276 contrast, Per1 and Per2, which provide negative feedback by interfering with the 277 Npas/Bmal1/Clock transcriptional complexes, are down-regulated, and Cry1 was 278 unchanged. In the bile duct ligation model of Per2-null mice Per2 played a protective 279 effects of cholestasis, atorvastatin repressed the expression of this feedback genes may 280 increase the risk of Liver injury (Chen et al. 2013). Thus, atorvastatin-induced disruption 281 of hepatic BA homeostasis was related to the core-clock gene alterations.

Dbp, Nr1d1 and Tef are direct clock controlled output genes. Circadian expression of HMG-CoA was disrupted in Rev-Erba (Nr1d1)-null mice and the circadian rhythms of liver Cyp7a1, SHP were decreased in Nr1d1-null mice (Beltowski et al. 2009). In the present study, the expression of $\mathrm{Nr} 1 \mathrm{~d} 1$ tended to decrease but was not significant. The PAR-domain basic leucine zipper (PAR bZip) transcription factors, Dbp and Tef, accumulate in a highly circadian manner in liver participating in cellular metabolism (Gachon et al. 2006). In the present study, the expression of both Dbp and Tef was decreased by repeated administration of atorvastatin. Ethanol was show to alter the peripheral clock, including Dbp and Tef, without altering the central SCN clock (Filiano et 
293 Ideally, circadian rhythms would be better illustrated using multiple time points as shown

294 in our recent publications with 6 time points (Li et al., 2016; Lu et al., 2013; Xu et al.,

295 2012; Zhang et al., 2012). However, circadian rhythms can also be evaluated using a

296 single time point as shown in our recent publications (Li et al., 2017). Thus, one time

297 point evaluation is also informative, depending on the goals and sample feasibility of the

298 study. In our preliminary study with 7-day atorvastatin administration, the upregulation of

299 Cyp7a1 and Bmal1 at multiple time points was observed (supplementary Figure 1).

300 In summary, repeated administration of atorvastatin altered bile acid metabolism and

301 disposition. Atorvastatin increased the expression of the bile acid synthesis rate-limiting 302 enzyme gene Cyp7a1, together with alterations in circadian clock genes. 303 Chronopharmacology has emerged as a new insight into the therapeutic use of drugs 304 (Dallmann et al. 2014; Ferrell \& Chiang 2015a; Li et al. 2016), and the effects of 305 atorvastatin on circadian clock is worthy of further investigation. 


\section{References}

Allen $\mathrm{K}$, Jaeschke $\mathrm{H}$, and Copple BL. 2011. Bile acids induce inflammatory genes in hepatocytes: a novel mechanism of inflammation during obstructive cholestasis. Am J Pathol 178:175186.

Alscher DM, Redmann D, Wehner F, Maier A, Mettang T, Kuhlmann U, and Fritz P. 2002. Metallothionein in liver-biopsies from patients with different diseases. Exp Toxicol Pathol 54:245-253.

Beltowski J, Wojcicka G, and Jamroz-Wisniewska A. 2009. Adverse effects of statins mechanisms and consequences. Curr Drug Saf 4:209-228.

Bjornsson E, Jacobsen El, and Kalaitzakis E. 2012. Hepatotoxicity associated with statins: reports of idiosyncratic liver injury post-marketing. J Hepatol 56:374-380.

Bjornsson ES. 2015. Drug-induced liver injury: an overview over the most critical compounds. Arch Toxicol 89:327-334.

Bozek K, Relogio A, Kielbasa SM, Heine M, Dame C, Kramer A, and Herzel H. 2009. Regulation of clock-controlled genes in mammals. PLoS One 4:e4882.

Byun HW, Hong EM, Park SH, Koh DH, Choi MH, Jang HJ, Kae SH, and Lee J. 2014. Pravastatin activates the expression of farnesoid $\mathrm{X}$ receptor and liver $\mathrm{X}$ receptor alpha in Hep3B cells. Hepatobiliary Pancreat Dis Int 13:65-73.

Chen P, Kakan X, Wang S, Dong W, Jia A, Cai C, and Zhang J. 2013. Deletion of clock gene Per2 exacerbates cholestatic liver injury and fibrosis in mice. Exp Toxicol Pathol 65:427-432.

Cheng X, Buckley D, and Klaassen CD. 2007. Regulation of hepatic bile acid transporters Ntcp and Bsep expression. Biochem Pharmacol 74:1665-1676.

Chiang JY. 2013. Bile acid metabolism and signaling. Compr Physiol 3:1191-1212.

Csanaky IL, Lu H, Zhang Y, Ogura K, Choudhuri S, and Klaassen CD. 2011. Organic aniontransporting polypeptide $1 \mathrm{~b} 2$ (Oatp1b2) is important for the hepatic uptake of unconjugated bile acids: Studies in Oatp1b2-null mice. Hepatology 53:272-281.

Dallmann R, Brown SA, and Gachon F. 2014. Chronopharmacology: new insights and therapeutic implications. Annu Rev Pharmacol Toxicol 54:339-361.

Ding Y, Zhao L, Huang ZH, Mei H, and Peng HM. 2008. [Role of early growth response factor-1 signal pathway in acute intrahepatic cholestatic hepatic injury in rats]. Zhonghua Gan Zang Bing Za Zhi 16:215-219.

Duez H, van der Veen JN, Duhem C, Pourcet B, Touvier T, Fontaine C, Derudas B, Bauge E, Havinga R, Bloks VW, Wolters H, van der Sluijs FH, Vennstrom B, Kuipers F, and Staels B. 2008. Regulation of bile acid synthesis by the nuclear receptor Rev-erbalpha. Gastroenterology 135:689-698.

Ferrell JM, and Chiang JY. 2015a. Circadian rhythms in liver metabolism and disease. Acta Pharm $\sin B$ 5:113-122.

Ferrell JM, and Chiang JY. 2015b. Short-term circadian disruption impairs bile acid and lipid homeostasis in mice. Cell Mol Gastroenterol Hepatol 1:664-677.

Filiano AN, Millender-Swain T, Johnson R, Jr., Young ME, Gamble KL, and Bailey SM. 2013. Chronic ethanol consumption disrupts the core molecular clock and diurnal rhythms of metabolic genes in the liver without affecting the suprachiasmatic nucleus. PLoS One 
8:e71684.

Fu ZD, Cui JY, and Klaassen CD. 2014. Atorvastatin induces bile acid-synthetic enzyme Cyp7a1 by suppressing FXR signaling in both liver and intestine in mice. J Lipid Res 55:2576-2586.

Gachon F, Olela FF, Schaad O, Descombes P, and Schibler U. 2006. The circadian PAR-domain basic leucine zipper transcription factors DBP, TEF, and HLF modulate basal and inducible xenobiotic detoxification. Cell Metab 4:25-36.

Grover HS, Luthra S, and Maroo S. 2014. Are statins really wonder drugs? J Formos Med Assoc 113:892-898.

Ikeda M, and Nomura M. 1997. cDNA cloning and tissue-specific expression of a novel basic helix-loop-helix/PAS protein (BMAL1) and identification of alternatively spliced variants with alternative translation initiation site usage. Biochem Biophys Res Commun 233:258264.

Jiang XY, Zhang Q, Chen P, Li SY, Zhang NN, Chen XD, Wang GC, Wang HB, Zhuang MQ, and Lu M. 2012. CYP7A1 polymorphism influences the LDL cholesterol-lowering response to atorvastatin. J Clin Pharm Ther 37:719-723.

Jurevics H, Hostettler J, Barrett C, Morell P, and Toews AD. 2000. Diurnal and dietary-induced changes in cholesterol synthesis correlate with levels of mRNA for HMG-CoA reductase. J Lipid Res 41:1048-1054.

Kalantari S, and Naghipour M. 2014. Statin therapy and hepatotoxicity: Appraisal of the safety profile of atorvastatin in hyperlipidemic patients. Adv Biomed Res 3:168.

Kerzner S, Irabagon N, and Berkelhammer C. 2013. Statin-induced cholestatic hepatitis: confirmed on rechallenge. Gastroenterol Hepatol (N Y) 9:603-605.

Kim ND, Moon JO, Slitt AL, and Copple BL. 2006. Early growth response factor-1 is critical for cholestatic liver injury. Toxicol Sci 90:586-595.

Klaassen CD, and Aleksunes LM. 2010. Xenobiotic, bile acid, and cholesterol transporters: function and regulation. Pharmacol Rev 62:1-96.

Kolouchova G, Brcakova E, Hirsova P, Sispera L, Tomsik P, Cermanova J, Hyspler R, Slanarova M, Fuksa L, Lotkova H, and Micuda S. 2011. Pravastatin modulates liver bile acid and cholesterol homeostasis in rats with chronic cholestasis. J Gastroenterol Hepatol 26:1544-1551.

Kovar J, Lenicek M, Zimolova M, Vitek L, Jirsa M, and Pitha J. 2010. Regulation of diurnal variation of cholesterol 7alpha-hydroxylase (CYP7A1) activity in healthy subjects. Physiol Res 59:233-238.

Le Martelot G, Claudel T, Gatfield D, Schaad O, Kornmann B, Lo Sasso G, Moschetta A, and Schibler U. 2009. REV-ERBalpha participates in circadian SREBP signaling and bile acid homeostasis. PLoS Biol 7:e1000181.

Lee SM, Zhang Y, Tsuchiya H, Smalling R, Jetten AM, and Wang L. 2015. Small heterodimer partner/neuronal PAS domain protein 2 axis regulates the oscillation of liver lipid metabolism. Hepatology 61:497-505.

Li H, Li WK, Lu YF, Wei LX, and Liu J. 2016. The Tibetan medicine Zuotai influences clock gene expression in the liver of mice. PeerJ 4:e1632.

Li H, Lu YF, Chen H, Liu J. 2017. Dysregulation of metallothionein and circadian genes in human hepatocellular carcinoma. Choronobiol Int 20, 1-11. 
391

Li MK, and Crawford JM. 2004. The pathology of cholestasis. Semin Liver Dis 24:21-42.

Liu J, Lu YF, Zhang Y, Wu KC, Fan F, and Klaassen CD. 2013. Oleanolic acid alters bile acid metabolism and produces cholestatic liver injury in mice. Toxicol Appl Pharmacol 272:816-824.

Lu YF, Jin T, Xu Y, Zhang D, Wu Q, Zhang YK, and Liu J. 2013. Sex differences in the circadian variation of cytochrome p450 genes and corresponding nuclear receptors in mouse liver. Chronobiol Int 30:1135-1143.

Ma K, Xiao R, Tseng HT, Shan L, Fu L, and Moore DD. 2009. Circadian dysregulation disrupts bile acid homeostasis. PLoS One 4:e6843.

Merli M, Bragazzi MC, Giubilo F, Callea F, Attili AF, and Alvaro D. 2010. Atorvastatin-induced prolonged cholestasis with bile duct damage. Clin Drug Investig 30:205-209.

Noshiro M, Usui E, Kawamoto T, Kubo H, Fujimoto K, Furukawa M, Honma S, Makishima M, Honma K, and Kato Y. 2007. Multiple mechanisms regulate circadian expression of the gene for cholesterol 7alpha-hydroxylase (Cyp7a), a key enzyme in hepatic bile acid biosynthesis. J Biol Rhythms 22:299-311.

Pan X, Zhang Y, Wang L, and Hussain MM. 2010. Diurnal regulation of MTP and plasma triglyceride by CLOCK is mediated by SHP. Cell Metab 12:174-186.

Parker RA, Garcia R, Ryan CS, Liu X, Shipkova P, Livanov V, Patel P, and Ho SP. 2013. Bile acid and sterol metabolism with combined HMG-COA reductase and PCSK9 suppression. J Lipid Res 54:2400-2409.

Perez MJ, and Briz O. 2009. Bile-acid-induced cell injury and protection. World J Gastroenterol 15:1677-1689.

Russo MW, Scobey M, and Bonkovsky HL. 2009. Drug-induced liver injury associated with statins. Semin Liver Dis 29:412-422.

Sullivan BP, Cui W, Copple BL, and Luyendyk JP. 2012. Early growth response factor-1 limits biliary fibrosis in a model of xenobiotic-induced cholestasis in mice. Toxicol Sci 126:267-274.

Wu N, Kim KH, Zhou Y, Lee JM, Kettner NM, Mamrosh JL, Choi S, Fu L, and Moore DD. 2016. Small Heterodimer Partner (NROB2) Coordinates Nutrient Signaling and the Circadian Clock in Mice. Mol Endocrinol 30:988-995.

Xu YQ , Zhang D, Jin T, Cai DJ, Wu Q, Lu YF, Liu J, Klaassen CD. Diurnal variation of hepatic antioxidant gene expression in mice. PLoS One. 2012;7(8):e44237. 2012.

Ye R, Selby CP, Chiou YY, Ozkan-Dagliyan I, Gaddameedhi S, and Sancar A. 2014. Dual modes of CLOCK:BMAL1 inhibition mediated by Cryptochrome and Period proteins in the mammalian circadian clock. Genes Dev 28:1989-1998.

Zhang D, Jin T, Xu YQ, Lu Y, Wu Q, Zhang YK, Liu J. Diurnal-and sex-related difference of metallothionein expression in mice. J Circadian Rhythms. 10(1):5, 2012.

Zhang YK, Guo GL, and Klaassen CD. 2011. Diurnal variations of mouse plasma and hepatic bile acid concentrations as well as expression of biosynthetic enzymes and transporters. PLoS One 6:e16683. 